\title{
Clinical Comparison of Treatments for Dentine Sensitivity from Non-carious Cervical Lesions
}

\author{
Karen Pintado-Palomino ${ }^{1}$, Bruna Neves de Freitas ${ }^{1}$, Larisse \\ Eduardo Adami ${ }^{1}$, Mariana Montanhini de Lima ${ }^{1}$, Takami Hirono \\ Hotta $^{1}$, Wilson Matsumoto ${ }^{1}$, Cesar Bataglion ${ }^{2}$ and Camila \\ Tirapelli $^{1 *}$ \\ ${ }^{1}$ Department of Dental Materials and Prosthodontics, School of Dentistry of \\ Ribeirão Preto, University of São Paulo, Brazil \\ ${ }^{2}$ Department of Restorative Dentistry, School of Dentistry of Ribeirão Preto, \\ University of São Paulo, Brazil \\ *Corresponding Author: Camila Tirapelli, Department of Dental Materials and \\ Prosthodontics, School of Dentistry of Ribeirão Preto, University of São Paulo, Brazil. \\ DOI: $10.31080 /$ ASDS.2020.04.0888
}

Received: June 30, 2020

Published: July 27, 2020

(C) All rights are reserved by Camila

Tirapelli, et al.

\begin{abstract}
Background: This study compared the effectiveness of in-office and at home treatments for cervical dentine sensitivity (CDS) from non-carious cervical lesions.

Methods: Sixty participants with CDS were selected and randomly allocated into six groups of treatment: LA: gallium aluminium arsenide laser and LP: laser-placebo (4 in-office sessions); RMGI: resin-modified glass-ionomer and ARC: adhesive resin cement (in-office single session); DD: stannous fluoride desensitizing dentifrice and RD: regular dentifrice (30 days at home-use). CDS was assessed with the visual analogue scale at pre-treatment, immediately after treatment and at 1, 2 weeks, 1 and 2 months after the end of treatment. Data were compared using the Kruskal-Wallis and Friedman tests ( $p<0.05)$.

Result: All treatments, except ARC, showed a significant CDS reduction at different times when compared to pre-treatment ( $\mathrm{p}<0.05)$. Resin-modified glass-ionomer, showed statistical differences $(\mathrm{p}<0.05)$ when compared to LA, LP and ARC groups immediately after treatment. Dentifrices (desensitizing and regular) showed statistical differences $(p<0.05)$ immediately after the 30 days of use.

Conclusion: All strategies, except ARC, were capable to reduce CDS at different times and maintain the relief up two months. Resinmodified glass-ionomers and dentifrices were the most effective strategies in reducing CDS just after the end of the proposed treatment.

Keywords: Dentine Sensitivity; Dentifrices; Effectiveness; Laser Therapy; Resin-Modified Glass-Ionomer
\end{abstract}

\section{Introduction}

Non-carious cervical lesions (NCCLs) are defined as the loss of hard tissue at cemento-enamel junction of the teeth that is not related to caries [1]. More recently, it has been estimated that the prevalence of NCCLs is around of $47 \%$ of population and higher in adult population [2] with higher frequency in maxilla and premolars [1]. Literature has suggested the multifactorial etiology of these lesions [1-3] which can result from wear friction, microstructural loss by occlusal loading and chemical degradation process [3].

As NCCLs involve a loss of enamel and dentin exposure at gingival margin, these lesions have been associated with the occurrence of cervical dentin sensitivity (CDS) [1]. CDS is a clinical condition characterized by a short, sharp pain in response to thermal, evaporative, tactile, chemical or osmotic stimuli [1]. These stimuli cause 
fluid movement in the dentinal tubule that excites mechanoreceptors in the periphery of the pulp and leads pain [4]. CDS is still commonly found in dental practice and it is of great interested because impacts quality life, hindering daily activities such as eating, drinking and tooth-brushing [5].

Besides the removal of etiological factor, the strategies for the treatment of CDS are based on two mechanisms of action: obliteration of the exposed dentine tubules and blocking neural activity that may be conducted in-office or at home [6]. In the obliteration of the dentin tubules approach, the strategies include the use of resin-based glass-ionomer, bonding agent, sealants as well as fluorides, oxalates, strontium, calcium phosphate, arginine, glutaraldehyde and bioglass containing products [7-13]. In the blocking neural approach, chemical potassium containing agents are commonly used due to their capability on blocking of impulse transmission achieved by potassium ions [8-14]. Also, low power laser device is a physical agent employed by the biomodularity effects on relief pain [8,15-17].

Despite the outcomes from clinical studies, evaluating diversity of products and approaches, support effectiveness in reducing CDS irrespective of mechanism of action [18], there is no a standardized treatment protocol in dental practice. Recently, the results of a meta-analysis [6] suggests that dentinal tubule occlusion (chemical or physical) and nerve desensitization provide the best outcomes for in-office treatment meanwhile only chemical dentin tubules and nerve desensitization for at home treatments. However, to best of our knowledge there are no clinical studies comparing in- office and at home strategies to manage symptomatic NCCLs.

Therefore, this study proposed to clinically compare the effectiveness of different in-office and at home strategies to treat CDS from NCCLs. The null hypothesis was there would be no differences among the tested treatments regarding effectiveness and duration on CDS reduction.

\section{Materials and Methods}

This study was a clinical study conducted from August 2017 to October 2018 that compared different strategies to treat CDS due to NCCLs in a two-month follow-up. It was approved by the local ethics committee, which follows the principles of the Declaration of Helsinki (protocol number $n^{\circ}$ 04969312.6.0000.5419). Before enrolment, each volunteer signed the term of consent from the ethics committee that included all the information regarding the risks and benefits of treatment. This study was registered on Clinical Trials, ID: 04969312.6.0000.5419.

\section{Sample size}

Sample size was calculated using G*Power 3.1 software [19] for an expected effect size of $20 \%$, an $\alpha$ level of 0.05 , a power of $80 \%$ and taking into account 06 groups, 06 assessments and a correspondent repeated assess of 0.5 . The calculation resulted in $n=54$ as the minimal total sample. Patients were considered as the unit of the study.

\section{Randomization}

The allocation of the groups was done using Microsoft Excel. In participants with CDS in more than one tooth, the tooth with the highest CDS according to the VAS was chosen as the test tooth. Thus, only one intervention per participant was done.

\section{Blinding}

The examiner involved in the CDS assessment was blind about the treatments applied. In the groups treated with the laser and its placebo, the participants and the examiner were blinded for the treatment, and the operator was aware of the treatment. In the groups treated with restorative treatment, the operator was aware of the treatment type, and the examiner and participant were unaware. In the groups treated with desensitizing and placebo dentifrices, the operator, examiner and the participants were unaware of the dentifrice delivered.

\section{Participants}

For enrolment, a member of the research staff examined the participants according to the eligibility criteria. To be included in the study, the participants should meet the inclusion criteria as follows: (a) male or female between 18 to 40 years old, seeking for dentin sensitivity treatment, (b) good general (no disease that implies intermittent use of drugs) and oral health (no alterations in the hard or soft tissues), (c) a minimum of 24 permanent teeth, (d) small (approximately $1 \mathrm{~mm}$ deep) NCCLs in at least one upper or lower canine, premolar or molar teeth and (e) confirmative diagnosis of CDS caused by a thermal or mechanical stimulus (subject responds to stimulus, considers stimulus to be painful, and requests discontinuation of the stimulus). 
The exclusion criteria for participating were: (a) chronic analgesic or anti-inflammatory therapy, (b) orthodontic treatment or dental prosthesis, (c) bleaching and therapy with desensitizing agent in the preceding 3 months, (d) periodontal disease and (e) women who were pregnant.
A total of 240 patients were assessed for eligibility, 180 were excluded, and 60 were included as total sample that were allocated to the study groups (Figure 1 - Flowchart).

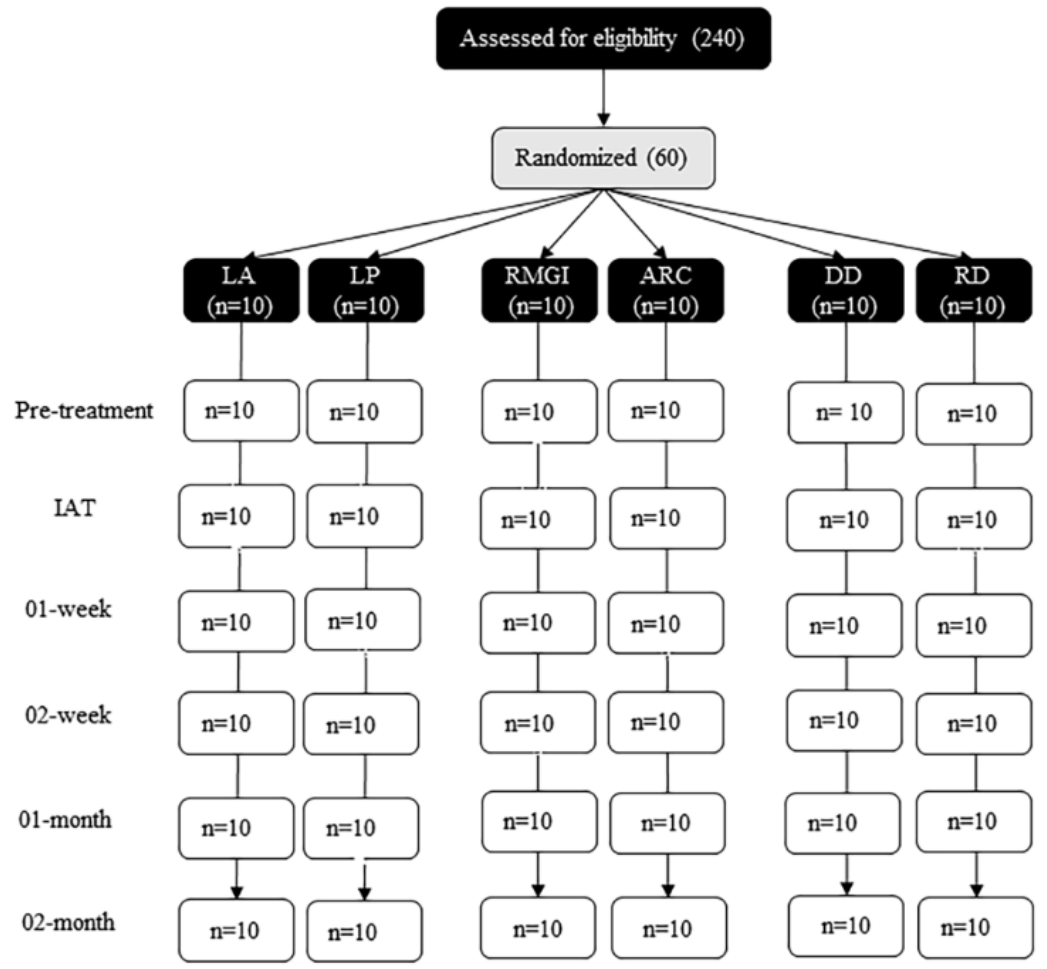

Figure 1: Flow chart of the study.

LA: Laser Group; LP: Laser Placebo Group; RMGI: Resin-Modified Glass-Ionomer Group; ARC: Adhesive Resin Cement; DD: Stannous Fluoride Desensitizing Dentifrice Group; RD: Regular Dentifrice; IAT: Immediately After Treatment

\section{Interventions}

A total of 60 participants were enrolled in the study and were randomly allocated into six groups $(n=10)$ according to the proposed treatments (Table 1):

- Laser (LA): The selected teeth received irradiation with a gallium aluminium arsenide (GaAlAs) laser (MM Optics, São Carlos, SP, Brazil) of $780 \mathrm{~nm}$ and $70 \mathrm{~mW}$ in four sessions with a
$72 \mathrm{~h}$ interval. It was applied to the cementum-enamel of the canine teeth (three points on the vestibular surface and one point on the lingual surface), molar or premolar teeth (two points on both vestibular and lingual surfaces). In the first session, laser irradiation was set at $6.0 \mathrm{~J} / \mathrm{cm}^{2}(0.25 \mathrm{~J}$ and 6 seconds per point), the next two sessions it was set at $2 \mathrm{~J} / \mathrm{cm}^{2}$ (0.2 J per point). 


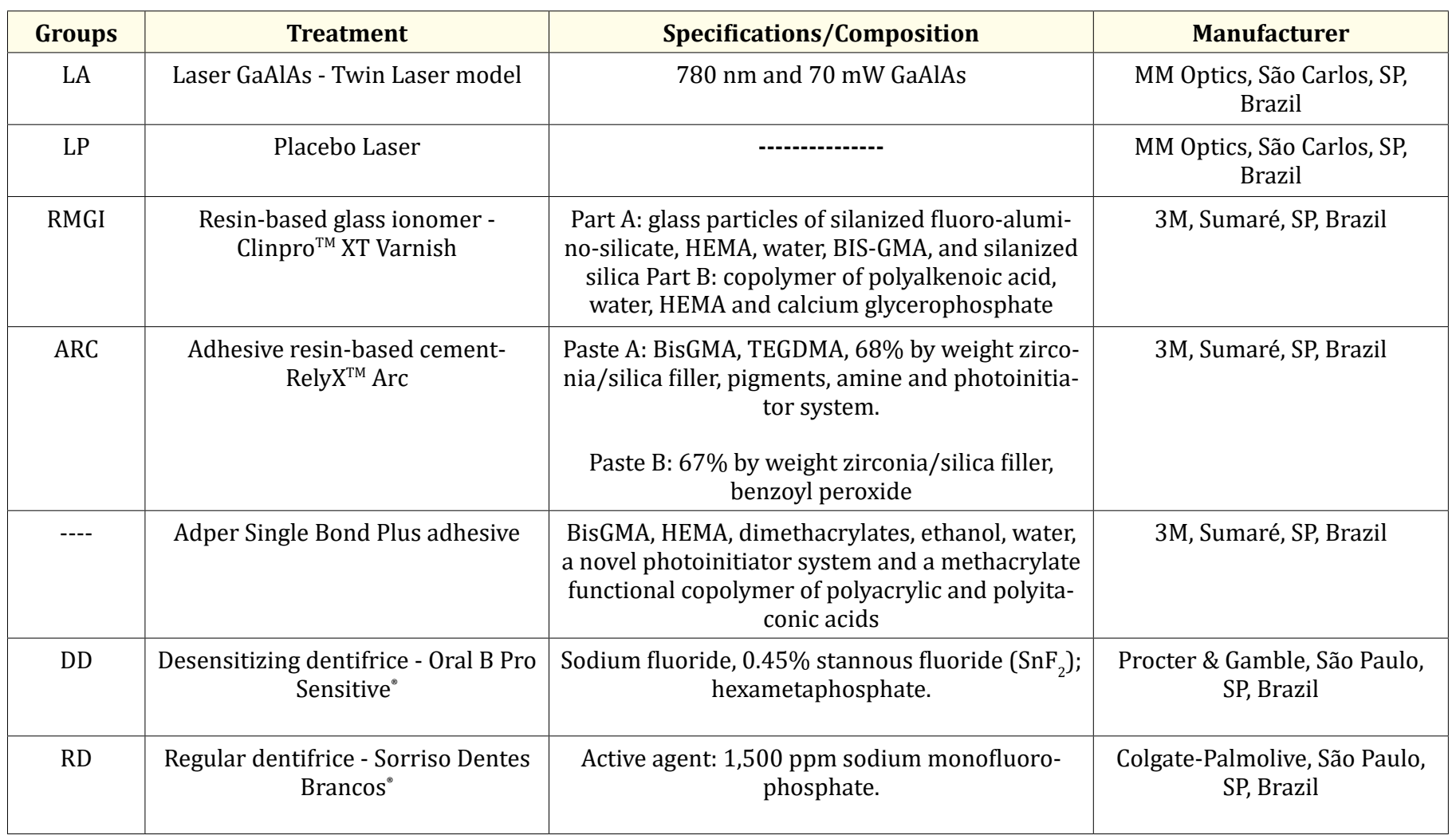

Table 1: Products used in the study.

- $\quad$ Laser placebo (LP): The same protocol as the LA group; however, the laser device used in this group did not have effective laser emission; it was only guided by light.

- $\quad$ Resin-modified glass-ionomer (RMGI): A single operator applied a thin layer of resin-modified glass-ionomer $(3 \mathrm{M}$, Sumaré, SP, Brazil) on the cervical surface of the affected teeth following the manufacturer's instructions. The product was light-cured for 20 seconds using a LED dental curing light unit (Lec Plus, MMO, Sao Carlos, SP, Brazil) at $700 \mathrm{~mW} / \mathrm{cm}^{2}$.

- $\quad$ Adhesive resin cement (ARC): The operator etched the NCCLs surface on enamel (20s) and dentin (10s), rinsed and blotted excess water using a toilet paper to apply 2 - 3 consecutive coats of the adhesive system (Adper Single Bond Plus Adhesive, 3M, Sumaré, SP, Brazil) with gentle agitation using a micro applicator. A gently air was applied to evaporate the solvent and light cured using a using a LED dental curing light unit (Lec Plus, MMO, Sao Carlos, SP, Brazil) at $700 \mathrm{~mW} / \mathrm{cm}^{2}$. Then, a thin layer of resin cement (RelyX ${ }^{\mathrm{TM}} \mathrm{Arc}$, 3M, Sumaré,
SP, Brazil) was applied on the same surface and light-cured for 20 seconds using a LED dental curing light unit (Lec Plus, MMO, Sao Carlos, SP, Brazil) at $700 \mathrm{~mW} / \mathrm{cm}^{2}$ Finally, excesses of resin cement was removed with diamond finishing burs (KG, Sorensen, Cotia, SP, Brazil).

- Desensitizing dentifrice (DD): The participants received a blinded dentifrice with $0.45 \%$ stannous fluoride (SnF2) (Procter and Gamble, São Paulo, SP, Brazil) and a soft-bristled manual toothbrush. In order to standardize the buccal hygiene, they were instructed about oral hygiene technique and to perform brushing three times a day for at least 2 to 3 minutes, for 30 days.

- $\quad$ Regular dentifrice (RD): The participants received a blinded dentifrice with 1500 ppm of sodium monofluorophosphate (Colgate-Palmolive, São Paulo, SP, Brazil) and the same soft-bristled manual toothbrush as the anterior group. The protocol used was similar to the one used in group DD. 


\section{Assessments}

The VAS assessments were taken at pre-treatment, immediately after the end of each treatment (IAT) and at 1-week, 2-weeks, 1-month and 2-months after the end of assigned treatments. In each assessment point, the CDS was assessed by a single examiner, who was not involved in the intervention, using the visual analogue scale (VAS) [20]. It consisted in a horizontal millimeter line, which extremities were marked by perpendicular bars ranged from 0 ("no pain" at the left) to 10 ("intolerable pain" at the right). The selected teeth were thermally stimulated by cold air at approximately $20^{\circ} \mathrm{C}$, from a triple syringe placed $3 \mathrm{~cm}$ from the buccal surface in the cervical area, perpendicular to the long axis of the tooth at full blast for $3 \mathrm{~s}$. In addition, they received a mechanical stimulus by a number 5 dental probe over the cervical area. The participants were instructed to record their response to the stimuli by placing a mark on the scale while the hypersensitivity test was carried out.

\section{Statistical analysis}

Comparisons between the treatment and the periods of evaluation were made using non-parametric tests, Kruskal-Wallis and Friedman tests, respectively, using the Prism 6.0 software (GraphPad, CA, USA).

\section{Results}

Sixty participants completed the study. The sample consisted of 42 females and 18 males, with a median age of 52 years (range 21 to 63 years). The teeth included were 3 upper canines, 15 upper first premolars, 12 upper second premolars, 6 upper first molars, 3 upper second molars, 11 lower first premolars, 5 lower second premolars, 2 lower first molars and 3 lower second molars. Table 2

\begin{tabular}{|l|c|c|c|c|c|c|}
\hline \multirow{2}{*}{\begin{tabular}{c}
\multirow{2}{*}{$\begin{array}{c}\text { Assessment } \\
\text { points }\end{array}$} \\
\cline { 2 - 7 }
\end{tabular}} & LA & LP & RMGI & ARC & DD & RD \\
\hline Pre-treatment & $5.0 \mathrm{a}, \mathrm{b}$ & $4.5 \mathrm{a}, \mathrm{b}$ & $6.0 \mathrm{a}, \mathrm{b}$ & $7.0 \mathrm{a}, \mathrm{b}$ & $4.0 \mathrm{a}, \mathrm{b}$ & $4.5 \mathrm{a}, \mathrm{b}$ \\
\hline IAT & $3.0 \mathrm{a}, \mathrm{b}$ & $2.0 \mathrm{a}, \mathrm{b}$ & $1.0 \mathrm{a}^{*}, \mathrm{~b}^{*}$ & $2.0 \mathrm{a}, \mathrm{b}$ & $0.6 \mathrm{a}^{*} \mathrm{~b}^{*}$ & $0.6 \mathrm{a}^{*} \mathrm{~b}^{*}$ \\
\hline 1-week & $1.0 \mathrm{a}, \mathrm{b}^{* *}$ & $1.5 \mathrm{a}, \mathrm{b}^{*}$ & $0.5 \mathrm{a}, \mathrm{b}^{* *}$ & $4.0 \mathrm{a}^{*}, \mathrm{~b}$ & $1.0 \mathrm{a}^{*}$ & $1.0 \mathrm{a}, \mathrm{b}^{*}$ \\
\hline 2-week & $1.0 \mathrm{a}, \mathrm{b}^{* *}$ & $1.5 \mathrm{a}, \mathrm{b}^{*}$ & $1.5 \mathrm{a}, \mathrm{b}^{* *}$ & $4.0 \mathrm{a}^{*}, \mathrm{~b}$ & $2.0 \mathrm{a}^{*}$ & $2.0 \mathrm{a}, \mathrm{b}^{*}$ \\
\hline 1-month & $1.0 \mathrm{a}, \mathrm{b}^{* *}$ & $2.0 \mathrm{a}, \mathrm{b}^{*}$ & $0.5 \mathrm{a}, \mathrm{b}^{* *}$ & $4.0 \mathrm{a} \mathrm{a}^{*} \mathrm{~b}$ & $0.5 \mathrm{a}, \mathrm{b}^{* *}$ & $0.5 \mathrm{a}, \mathrm{b}^{* *}$ \\
\hline 2-month & $1.0 \mathrm{a}, \mathrm{b}^{* *}$ & $2.0 \mathrm{a}, \mathrm{b}^{*}$ & $0.5 \mathrm{a}, \mathrm{b}^{* *}$ & $4.0 \mathrm{a}^{*}, \mathrm{~b}$ & $0.5 \mathrm{a}^{*} \mathrm{~b}^{*}$ & $0.5 \mathrm{a}, \mathrm{b}^{* *}$ \\
\hline
\end{tabular}

Table 2 - Median for CDS in the assessment points according the VAS value.

$\dagger$ : In accordance with Kruskal-Wallis and Friedman tests $(\mathrm{p}<0.05)$, letter A/a indicates a statistical difference between the treatments in the same assessment point (row); letter B/b indicates a statistical difference between each treatment when the VAS scores after treatment were compared with the pre-treatment (column). ${ }^{*}=p<0.05 ;{ }^{* *}=p<0.01$ and ${ }^{* * *}=p<0.001$.

shows the CDS median values and statistical significance obtained according the treatment used and the assessment point.

Regarding the evaluation of CDS over the period of study, each treatment group was analyzed independently. The results indicated statically significant difference in all groups when compared to the pre-treatment $(\mathrm{p}<0.05)$, except ARC group that showed no statistically significant difference at any assessment point ( $p$ $>0.05$ ). The CDS was significantly reduced immediately after the in-office, single session treatment for RMGI ( $<<0.05)$. On DD ( $<<$ $0.05)$ and $R D(p<0.05)$ CDS was significantly reduced immediately after the 30 days of at home use treatment. For the LA ( $p<0.01)$, LP $(p<0.05)$ groups, CDS was significantly reduced at 1 -week after the end of the treatment. In these groups, the reduction of CDS was remained up two months after the end of each treatment.

Regarding the comparison of CDS among the groups at each assessment point, the results showed no significant difference at pretreatment assessment point ( $\mathrm{p}>0.05)$. CDS values were of medium intensity, indicating the homogeneity of the sample groups. At IAT assessment point, the RMGI, DD and DR groups had the lowest CDS median values showing a statistically significant difference $(\mathrm{p}<$ 
0.05) when compared to the LA, LP and ARC groups. At following assessment points (1-week, 2-week, 1-month and 2-months of the end of treatments), a significant difference was observed between ARC group, which presented the highest CDS median values, and all tested groups $(\mathrm{p}<0.05)$.

\section{Discussion}

The present study compared the effectiveness of different strategies to treat CDS form NCCLs. This study rejected the null hypothesis since the results showed that there is a difference among treatments used for CDS caused by NCCLs.

Regarding each treatment group over the period of the study, the evaluation of CDS revealed that RMGI, DD and DR groups were effective in CDS reduction just after the end of treatment proposed. In the following assessment point, performed at 1-week after the end of treatment, all groups showed to be efficient in reducing CDS and maintaining the relief up two months of the end of treatments with no significant differences over time, except ARC group that showed no decrease in pain throughout the period of study.

In this current study the product used in the in-office RMGI group was the Clinpro XT Varnish, a resin-modified glass-ionomer that mechanism of action is based on the dentinal obliteration. Previous in vitro studies have demonstrated the reduction in dentin permeability [21-23] with decrease of fluid flow around of $40-50 \%$ even after a single application of Clinpro XT [21] which could explain the fast relief of pain obtaining in this study. Also, in in vitro conditions, this desensitizing product demonstrated that is capable of preventing the reopening of the tubules after the erosive/abrasive challenges [21,23].

In fact, these in vitro results have been attributed to the chemical adhesion to dentin due to the methacrylate-modified polyalkenoic acid technology [24]. Besides of the chelation mechanism produced by this product, it has been suggested that Clinpro XT Varnish also releases calcium, phosphate and fluoride during erosive challenges and when in contact with saliva, promoting remineralization [23].

All these benefit characteristics could explain the immediate and lasting relief of the CDS obtained in our study. Moreover, clinical studies $[8,25,26]$ have corroborated the effectiveness of the resin-modified glass-ionomer in CDS immediately after application and over a period of up 6 months of follow-up, supporting our findings.

In regard to the at home DD and RD, both groups also showed to be efficient just after the end of the treatment and over the following assessment points. Dentifrices are the most common at home oral product and have become an important vehicle for the delivery of desensitizing agents based on dentine tubule obliteration or blocking impulse transmission in dentinal nerves. Stannous fluoride $\left(\mathrm{SnF}_{2}\right)$, the main active ingredient used in DD group, acts with tooth surfaces to produce insoluble precipitates into the intertubular dentin and dentinal tubules contributing to the reduction of dentin permeability and dentin tubule occlusion [13]. Also, $\mathrm{SnF}_{2}$ acts by facilitating the absorption of fluoride, interfering in the relationship between remineralisation and demineralization [27-30]. While some clinical studies showed the brushing [31] or the direct application [32] with $\mathrm{SnF}_{2}$ dentifrice can reduce CDS immediately and with continued use at short-term, others have been inconclusive especially at early time points [12]. Even though the results obtained in this study provides some evidence of the effectiveness on reducing CDS for the DD group, no difference was observed when compared to RD group at each assessment point. The regular dentifrice used in RD group was not designed for CDS treatment; however, the reduction in CDS suggests that this dentifrice, which contains 1500 ppm of sodium monoflurophosphate, can also provide effectiveness in relief of CDS in the same manner. Besides, the IAT assessment point in DD and DP groups were after 30 days of home-use of the dentifrices.

Concerning LA and LP group, the results showed the effectiveness in reducing CDS only at one-week after the end of the four in-office session treatment and it remained up two months. In this study, we used a low-intensity GaAlAs laser wavelength $(780 \mathrm{~nm})$, which mechanism of action is based on biological modulatory effects. It is postulated that this type of low output power lasers mediates an analgesic effect related to depressed nerve transmission [33]. It seems that the light emitted by the low-intensity laser stimulate the cell activities that promote the concentration of calcium, potassium and sodium ions and blocked the neural depolarization, providing analgesia and anti-inflammatory effects [34]. The effec- 
tiveness of low power laser in reducing CDS has been reported previously in clinical studies at short $[17,35]$ and long-term $[8,16,36]$. In these clinical studies different methodologies have been tested such as the type of dose and power, number of sessions and time interval and time of laser appliance resulting in a satisfactory reduction of $\mathrm{CDH}$, although it seems that a higher dose [36] and time exposure [35] were more efficient.

On the other hand, no significant difference between LA and LP was observed in this study. The placebo effect in the clinical studies treating dentine hypersensitivity has been shown corroborating our findings $[6,9,18]$. The reason for the placebo effect on the treatment of NCCLs could be related to the fact that the patient is participating in a study inside a reference center of research. The gained attention and information about their condition may have led to a shift in patient behavior that contributed to an improved oral health condition [33].

In regard to ARC group, the results revealed no significant reduction of CDS was occurred over course of study. The CDS treatment with an adhesive resin cement was not previously reported; however, the use of resin-based materials in NCCL are described in the literature [38-40]. We tested a conventional dual-cure resin cement as a physical barrier to prevent the occurrence of CDS because of the low solubility, high mechanical quality and adhesive properties [41,42], taking into account the fine pellicle of the material required for the small NCCLs of this study.

Although a reduction of CDS was reported by the patients in ARC group immediately after the in-office single session treatment, it was not significant when compared to pretreatment. Following assessment points no significant variations were also observed. This finding could be attributed to the pretreatment procedure on tooth surface required prior to placement of the resin-based covering that include an etching with phosphoric acid. Even though it was tried all steps were performed carefully, is probably the sensitive technique of etch and rinse system may have been caused residual sensitivity. Moreover, the lack of CDS reduction in ARC group, represented by the highest values over the study, justifies the significant difference found when compared to all tested groups and the less effectiveness.
In this study different at home and in-office strategies to treat CDS were tested. The results of this study suggests that at home and in-office treatment that acts by dentinal tubules obliteration is more effective and faster on CDS reduction. A systematic review [18] that compared the treatments used to treat dentin hypersensitivity concluded that irrespective of the mechanism of action, they are efficient in dentin hypersensitivity reduction in different follow-up times. According to this systematic review, only in-office treatments are effective in immediate reduction, maintaining its effectiveness over time and for long-time effects, at home treatments can also be efficient.

Among the limitations of this study, literature [18] has recommended a split-mouth design should be chosen whenever possible; however, participants did not meet the clinical conditions for this design. Also, a control group with placebo is desired in clinical studies evaluating efficacy of treatments. Despite the effort to accomplish this requirement, only a placebo for laser was performed. In the case of dentifrices groups, although the regular dentifrice used in RD group is not considering as a desensitizing agent, the fluoride content (1500 ppm, the minimum content allowed in the country where study was conducted) in the formulation may also interfere in CDS. Furthermore, comparison among treatments was some limited due to the modality of treatment. In at home treatment with dentifrices, instructions were given about brushing technique and their frequency; however, the home use treatment relied on the patient's compliance to work and may not always provide a reliable evaluation of their effects. Also, it is important to emphasize that the multifactorial etiology of CDS turns the success of treatment more complex. So, the correct diagnosis and the control of the etiological factor is required.

Thus, futures studies focusing in this topic would be necessary to better understanding the best choice of treatment.

\section{Conclusion}

According to the objectives of the study and considering its limitations, it is possible to conclude that: 
- $\quad$ All strategies of treatments were capable to reduce CDS at different times and maintain the relief up two months, except ARC.

- The in-office treatment with resin-modified glass-ionomer and at home dentifrices were the most effective and faster treatments in reducing CDS caused by NCCL.

\section{Acknowledgments}

The authors are grateful to CNPq (National Council of Technological and Scientific Development), CAPES (Coordination for the Improvement of Higher Education Personnel) and São Paulo Research Foundation, FAPESP: 12/08312-9; 11/07039-4; 11/19567-5; 10/12032-6.

\section{Conflict of Interest}

The authors have nothing to disclose.

\section{Bibliography}

1. Yoshizaki KT., et al. "Clinical features and factors associated with non-carious cervical lesions and dentin hypersensitivity". Journal of Oral Rehabilitation 44 (2017): 112-118.

2. Teixeira DNR., et al. "Prevalence of noncarious cervical lesions among adults: A systematic review". Journal of Dentistry 95 (2020): 103285.

3. Igarashi Y., et al. "The prevalence and morphological types of non-carious cervical lesions (NCCL) in a contemporary sample of people”. Odontology 105 (2017): 443-452.

4. Brannstrom M. "Sensitivity of dentine". Oral Surgery Oral Medicine Oral Pathology 2 (1996): 517-526.

5. Mason S., et al. "Impact of toothpaste on oral health related quality of life in people with dentine hypersensitivity". BMC Oral Health 19 (2019): 226.

6. Moraschini V., et al. "Effectiveness for dentine hypersensitivity treatment of non-carious cervical lesions: a meta-analysis". Clinical Oral Investigations 22 (2018): 617-631.

7. Celik EU., et al. "Three-year clinical evaluation of high-viscosity glass ionomer restorations in non-carious cervical lesions: a randomised controlled split-mouth clinical trial”. Clinical Oral Investigations 23 (2019): 1473-1480.
8. Moura GF., et al. "Four session Protocol Effectiveness in Reducing Cervical Dentin Hypersensitivity: A 24-Week Randomized Clinical Trial". Photobiomodulation, Photomedicine, and Laser Surgery 37 (2019): 117-123.

9. Tirapelli C., et al. "The effect of a novel crystallised bioactive glass-ceramic powder on dentine hypersensitivity: a long-term clinical study". Journal of Oral Rehabilitation 38 (2011): 253262.

10. Gillam DG., et al. "Comparison of two desensitizing agents for the treatment of cervical dentine sensitivity". Endodontics and Dental Traumatology 13 (1997): 36-39.

11. Guentsch A., et al. "Biomimetic mineralization: long-term observations in patients with dentin sensitivity". Dental Materials 28 (2012): 457-464.

12. Parkinson CR., et al. "Three randomized clinical trials to assess the short-term efficacy of anhydrous $0.454 \% \mathrm{w} / \mathrm{w}$ stannous fluoride toothpastes for the relief of dentin hypersensitivity". American Journal of Dentistry 29 (2016): 25-32.

13. Takamizawa T., et al. "Laboratory evaluation of dentin tubule occlusion after use of dentifrices containing stannous fluoride”. Journal of Oral Sciences 61 (2019): 276-283.

14. Freitas S., et al. "Dentin hypersensitivity treatment of non-carious cervical lesions -a single-blind, split-mouth study". Brazilian Oral Research 29 (2015): 45.

15. Femiano F., et al. "Effectiveness on oral pain of $808 \mathrm{~nm}$ diode laser used prior to composite restoration for symptomatic noncarious cervical lesions unresponsive to desensitizing agents". Lasers in Medical Science 32 (2017): 67-71.

16. Lopes AO., et al. "Evaluation of different treatment protocols for dentine hypersensitivity: an 18-month randomised clinical trial”. Lasers in Medical Science 32 (2017): 1023-1030.

17. Dantas EM., et al. "Clinical Efficacy of Fluoride Varnish and Low-level Laser Radiation in Treating Dentin Hypersensitivity". Brazilian Dental Journal 27 (2016): 79-82. 
18. Marto CM., et al. "Evaluation of the efficacy of dentin hypersensitivity treatments- A systematic review and follow-up analysis". Journal of Oral Rehabilitation 46 (2019): 952-990.

19. Faul F., et al. "G*Power 3: a flexible statistical power analysis program for social, behavioural, and biomedical sciences". Behavior Research Methods 39 (2007): 175-191.

20. Rosas S., et al. "Comparison between the Visual Ana$\log$ Scale and the Numerical Rating Scale in the perception of esthetics and pain". International Orthodontics 15 (2017): 543-560.

21. Terenzi M., et al. "Effectiveness of Clinpro XT in Reducing Dentin Permeability and its Resistance to Acid Challenges". Oral Health and Preventive Dentistry 16 (2018): 339-344.

22. Machado AC., et al. "Effect of in office desensitizers containing calcium and phosphate on dentin permeability and tubule occlusion". Journal of Dentistry 86 (2019): 53-59.

23. Garofalo SA., et al. "In Vitro Effect of Innovative Desensitizing Agents on Dentin Tubule Occlusion and Erosive Wear". Operative Dentistry 44 (2019): 168-177.

24. Virupaxi SG., et al. "Comparative evaluation of longevity of fluoride release from three different fluoride varnishes: an in vitro study". Journal of Clinical and Diagnostic Research 10 (2016): ZC33-ZC36.

25. Madruga MM., et al. "Evaluation of dentin hypersensitivity treatment with glass ionomer cements: A randomized clinical trial”. Brazilian Oral Research 5 (2017): 31:e3.

26. Canali GD., et al. "Clinical efficacy of resin-based materials for dentin hypersensitivity treatment". American Journal of Dentistry 30 (2017): 201-204.

27. Muhler JC., et al. "Studies on stannous fluoride and other fluorides in relation to the solubility of enamel in acid and the prevention of experimental dental caries". Journal of Dental Research 33 (1954): 33-49.

28. Algarni AA., et al. "The impact of stannous, fluoride ions and its combination on enamel pellicle proteome and dental erosion prevention". Public Library of Science One 10 (2015): e0128196.
29. Cheng X., et al. "Comparative effect of a stannous fluoride toothpaste and a sodium fluoride toothpaste on a multispecies biofilm". Archives of Oral Biology 74 (2017): 5-11.

30. Cvikl B., et al. "Stannous chloride and stannous fluoride are inhibitors of matrix metalloproteinases". Journal of Dentistry 79 (2018): 51-58.

31. Creeth J., et al. "Randomised clinical studies investigating immediate and short-term efficacy of an occluding toothpaste in providing dentine hypersensitivity relief". BMC Oral Health 4 (2019): 98.

32. Creeth J., et al. "Three randomized studies of dentine hypersensitivity reduction after short-term SnF2 toothpaste use". Journal of Clinical Periodontology 46 (2019): 1105-1115.

33. Kimura Y., et al. "Treatment of dentine hypersensitivity by lasers: a review". Journal of Clinical Periodontology 27 (2000): 715-721.

34. Gerschman JA., et al. "Low level laser therapy for dentinal tooth hypersensitivity". Australian Dental Journal 39 (1994): 353355.

35. Hashim NT., et al. "Effect of the clinical application of the diode laser $(810 \mathrm{~nm})$ in the treatment of dentine hypersensitivity". BMC Research Notes 7 (2014): 31.

36. Naghsh N., et al. "Evaluation of the Effects of 660-nm and 810nm Low-Level Diode Lasers on the Treatment of Dentin Hypersensitivity". Journal of Lasers in Medical Sciences 11 (2020): 126-134.

37. Maximiano V., et al. "Nd: YAG laser and calcium sodium phosphosilicate prophylaxis paste in the treatment of dentine hypersensitivity: a double-blind randomised clinical study". Clinical Oral Investigations 23 (2019): 3331-3338.

38. Szesz A., et al. "Effect of flowable composites on the clinical performance of non-carious cervical lesions: a systematic review and meta-analysis". Journal of Dentistry 65 (2017): 11-21.

39. Canali CD., et al. "One-year clinical evaluation of bulk-fill flowable vs regular nano-filled composite in non-carious cervical lesions". Clinical Oral Investigations 23 (2019): 889-897. 
40. Oz FD., et al. "An 18-month clinical evaluation of three different universal adhesives used with a universal flowable composite resin in the restoration of non-carious cervical lesions". Clinical Oral Investigations 23 (2019): 1443-1452.

41. Nocca G., et al. "Effects of barriers on chemical and biological properties of two dual resin cements". European Journal of Oral Sciences 123 (2015): 208-214.

42. Ladha K and Verma M. "Conventional and contemporary luting cements: an overview". Journal of Indian Prosthodontic Society 10 (2010): 79-88.

\section{Assets from publication with us}

- Prompt Acknowledgement after receiving the article

- Thorough Double blinded peer review

- Rapid Publication

- Issue of Publication Certificate

- High visibility of your Published work

Website: www.actascientific.com/

Submit Article: www.actascientific.com/submission.php

Email us: editor@actascientific.com

Contact us: +919182824667 